\title{
Fixed points of the Similarity Renormalization Group and the Nuclear Many-Body Problem
}

\author{
Presented by E. R. A. at 22th European Conference On Few-Body Problems In Physics: EFB22 \\ 9 - 13 Sep 2013, Krakow (Poland)
}

\begin{abstract}
The Similarity Renormalization Group reduces the off-shellness by driving the evolved interaction towards a diagonal band. We analyze the infrared limit and the corresponding on-shell interactions and its consequences for light nuclei. Using a harmonic oscillator shell model we obtain a Tjon line $B_{\alpha}=4 B_{t}-3 B_{d}$ which can be understood from a combinatorics counting of nucleon pairs and triplets in the triton and $\alpha$ particle and compares favorably with realistic calculations.
\end{abstract}

Keywords Similarity Renormalization Group · Few body problem · Off-shell

\section{Introduction}

Nuclear Physics has always been plagued with difficulties and ambiguities related to off-shellness of two-body forces and the inclusion of many-body forces deduced from the existence of equivalent Hamiltonians [1]. In a remarkable paper Glöckle and Polyzou [2] point out that both problems are actually intertwined since "different off-shell extensions of two-body forces can be equivalently realized as three-body interactions" and while "there are no experiments measuring only three-body binding energies and phase shifts that can determine if there are no three-body forces in a three-body system" it is still likely that "there may be some systems for which it is possible to find a representation in which three-body forces are not needed".

However, triton and $\alpha$-particle ab initio calculations (see e.g. [3] for a review) using 2-body high quality interactions, i.e. constrained to fit $\mathrm{NN}$ scattering data with $\chi^{2} /$ d.o.f $\lesssim 1$ display exceedingly simple regularities, such as the Tjon line, a linear correlation between the binding energies, $B_{\alpha}=a B_{t}+b$ within a relatively wide range of energies (see Fig. 2). This suggests the onset of some scale invariance [4] and the understanding of this correlation should also provide a credible value for the slope of the Tjon line.

Actually, there is much freedom in making unitary transformations keeping the two body bound and continuum spectrum while generating a wide range of three- and four-body properties. A practical way to generate these transformations is by means of the similarity renormalization group (SRG) proposed by Głazek

E.R.A. was supported by Spanish DGI (grant FIS2011-24149) and Junta de Andalucía (grant FQM225). S.S. was supported by FAPESP and V.S.T. by FAEPEX and CNPq. Computational power provided by FAPESP grants 2011/18211-2 and 2010/50646-6.

E. Ruiz Arriola

Departamento de Fisica Atómica, Molecular y Nuclear and Instituto Carlos I de Fisica Teórica y Computacional, Universidad de Granada, E-18071 Granada, Spain. E-mail: earriola@ugr.es

S. Szpigel

Faculdade de Computação e Informática, Universidade Presbiteriana Mackenzie, São Paulo, Brazil. E-mail: szpigel@mackenzie.br

V. S. Timóteo

Grupo de Óptica e Modelagem Numérica-GOMNI, Faculdade de Tecnologia, Universidade Estadual de Campinas-UNICAMP, Limeira, Brazil. E-mail: varese@ft.unicamp.br 
and Wilson [5] and independently by Wegner [6]. The SRG was first used within Nuclear Physics by Bogner, Furnstahl and Perry [7]. We discuss how the above mentioned regularities could be understood within a SRG context by analysing its infrared fixed points as we discussed previously [8]. The proposed scenario is free of ambiguites but indicates a predominant role played by 3-body forces even in triton and alpha nuclei.

\section{SRG evolution and the fixed points}

The general SRG equation corresponds to a one-parameter operator evolution dynamics given by [9],

$$
\frac{d H_{s}}{d s}=\left[\left[G_{s}, H_{s}\right], H_{s}\right]
$$

and supplemented with an initial condition at $s=0, H_{0}$. With the exception of some few cases [10, 11; 12$]$ these equations are mostly solved numerically [13], by implementing a high momentum UV cut-off, $\Lambda$, and an infrared momentum cut-off $\Delta p$, which reduces the analysis to the finite $N$-dimensional case with $\Lambda=N \Delta p$. The isospectrality of the SRG becomes evident from the trace invariance $\operatorname{Tr}\left(H_{s}\right)^{n}=\operatorname{Tr}\left(H_{0}\right)^{n}$.

Fixed points of Eq. (1) are given by stationary solutions, $\left[\left[G_{s}, H_{s}\right], H_{s}\right]=0$ requiring $\left[G_{s}, H_{s}\right]=F\left(H_{s}\right)$. Thus there exists a basis where both $G_{s}$ and $H_{s}$ can be simultaneously block-diagonal for different energy subspaces with dimension equal the degeneracy. The question is what choices of $G_{s}$ actually drive the solution to this block-diagonal form. We will assume the usual separation $H_{0}=T+V$. For generators which have the property $d / d s\left(\operatorname{Tr} G_{s}^{2}\right)=0$, and using cyclic properties of the trace and the invariance of $\operatorname{Tr}\left(H_{s}\right)^{n}$ one gets

$$
\frac{d}{d s} \operatorname{Tr}\left(H_{s}-G_{s}\right)^{2}=-2 \operatorname{Tr}\left(i\left[G_{s}, H_{s}\right]\right)^{\dagger}\left(i\left[G_{s}, H_{s}\right]\right) \leq 0 .
$$

Because $\operatorname{Tr}\left(H_{s}-G_{s}\right)^{2}$ is positive but its derivative is negative the limit for $s \rightarrow \infty$ exists and corresponds to the fixed points, i.e. any starting $H_{0}$ gets indeed diagonalized by the SRG equations (1). Thus, the SRG equation are just a continuous way of diagonalizing $H_{0}$. The key point is that there are many ways to diagonalize a Hamiltonian. For an $\mathrm{N}$-dimensional space there are $N$ ! possible permutations regarding the final ordering of states. There are two complementary generator choices: $G_{s}=H_{D}=\operatorname{diag}\left(H_{s}\right)$ by Wegner [6] and by Głazeck and Wilson [5] $G_{s}=T$. More generally $G_{s}=F(T)$ [14], describes a different trajectory but identical fixed points. A choice in between is the block-diagonal generator $G_{s}=P H_{s} P+Q H_{s} Q$ in two complementary subspaces with $P+Q=1$ [15, 16]. Denoting by $E_{n}$ the Hamiltonian and $\varepsilon_{n}$ the kinetic energy eigenvalues, the stability analysis [8] can be extended to show that $\left(C_{n m}\right.$ is some suitable matrix encoding the initial $\left.H_{0}\right)$

$$
H_{n m}(s)=E_{n} \delta_{n, m}+C_{n m}\left\{\begin{array}{lc}
e^{-\left(\varepsilon_{n}-\varepsilon_{m}\right)\left(E_{n}-E_{m}\right) s}+\ldots & \text { Wilson } \\
e^{-\left(E_{n}-E_{m}\right)^{2} s}+\ldots & \text { Wegner }
\end{array}\right.
$$

Therefore, while all $N$ ! fixed points are stable in the Wegner case, in the Wilson case there is a unique fixed point where the Hamiltonian preserves the original ordering of states according to the kinetic energy since $\left(\varepsilon_{n}-\varepsilon_{m}\right)\left(E_{n}-E_{m}\right)>0$. The emerging crossing structure will be discussed in more detail in Ref. [17].

The previous considerations answer the question of how similar are two given Hamiltonians in terms of the Frobenius scalar product, norm and induced metric,

$$
\langle A, B\rangle=\operatorname{Tr}\left(A^{\dagger} B\right), \quad\|V\|^{2} \equiv \operatorname{Tr}\left(V^{2}\right), \quad d(A, B) \equiv\|A-B\| .
$$

Moreover, because for $G_{s}=T$ the quantity $\operatorname{Tr}\left(V_{s}^{2}\right)$ is non-negative and decreasing, we have an interesting variational property of the Wilson generator, namely

$$
\lim _{s \rightarrow \infty} \operatorname{Tr}\left(V_{s}^{2}\right)=\left.\min _{V} \operatorname{Tr}(V)^{2}\right|_{T+V=U H_{0} U^{\dagger}} .
$$

Thus, the Wilson SRG drives the potential to the smallest, in the Frobenius norm sense, possible one with the same spectrum. In the continuum spectrum case, the orbital degeneracy induced by the $d^{3} p$ integration measure in the Frobenius norm has two complementary effects: i) It suppresses low energy states and ii) it enhances high energy components. Thus, minimizing $\|V\|$ along the SRG evolution transfers very efficiently high energy components into small ones, and thus softens the possible cores. This is the main reason why 

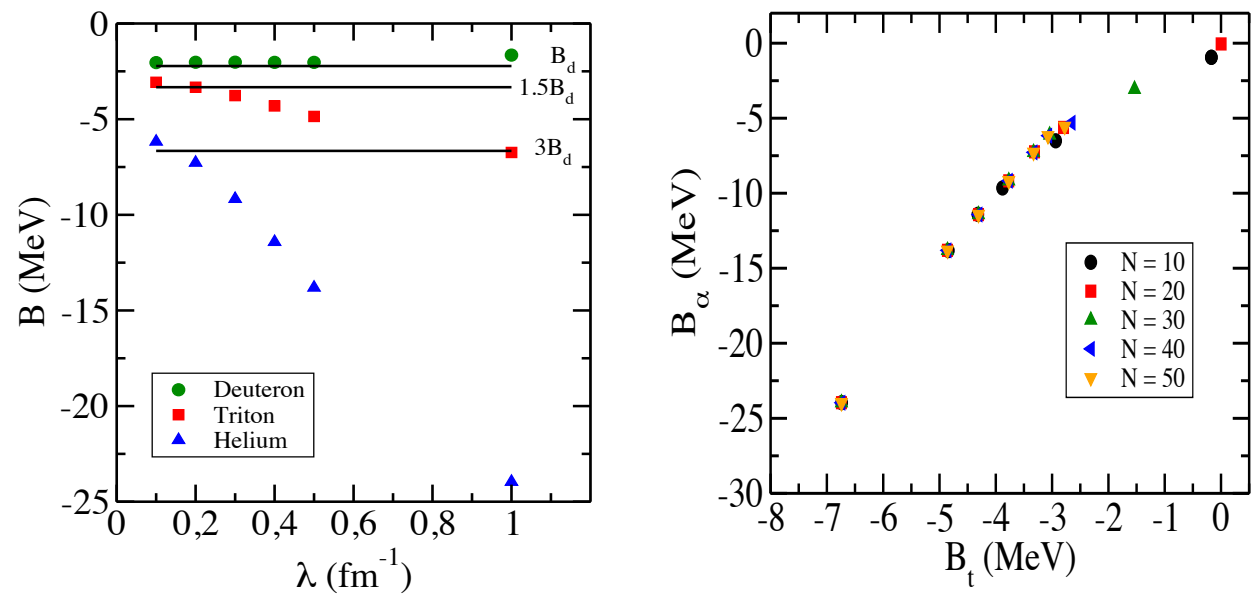

Fig. 1 Variational Shell Model Binding Energies (in $\mathrm{MeV}$ ) for different values of the SRG cut-off compared to the limiting on-shell values for $\lambda \rightarrow 0$ (left panel). $B_{\alpha}-B_{t}$ Tjon line for different $\mathrm{N}$ values of the momentum grid (right panel). In both cases we assume 3- and 4-body forces to vanish at that scale $\lambda, V_{3}(\lambda)=V_{4}(\lambda)=0$. The 2-body potentials $V_{2}(\lambda)$ are phase equivalent.

this approach became popular in Nuclear Physics where the SRG cut-off $\lambda=1 / s^{\frac{1}{4}}$ with energy dimensions is used [7]. In the many-body case where $H=T+V_{2}+V_{3}+V_{4}+\ldots$ one subtracts CM motion, $G_{s}=T_{\text {rel }} \equiv$ $T-T_{\mathrm{CM}}$ to preserve translational invariance and derive a hierarchy of induced n-body SRG equations. Note that the SRG method does not tell what the initial condition for the many body Hamiltonian should be, and this amounts to switch-on many body forces at any value of $\lambda$. Of course, once they are considered at any given $\lambda$ the isospectrality guarantees that the n-body energy remains independent of $\lambda$. This is checked for $n=3$ where the triton binding energy is fixed with moderate bare three body forces, $V_{3}(\lambda \rightarrow \infty)[18 ; 19 ; 20 ; 21,22 ; 23 ; 24]$.

Driving the potential to its diagonal form has the further advantage of reducing calculations to first order perturbation theory and also to get rid of the off-shell effects completely. For instance, the $R$-matrix on the momentum grid $p_{n}$ reduces to the on-shell potential of the Lippmann-Schwinger equation; for the ${ }^{1} S_{0}$ state $\lim _{\lambda \rightarrow 0} V_{\lambda}\left(p_{n}, p_{n}\right)=-\tan \delta^{\mathrm{LS}}\left(p_{n}\right) / p_{n}$ is a stable fixed point [8]. We have checked these trends regarding the fixed points numerically. Here, we illustrate our points using the Wilson generator although many features are common to the Wegner generator. Further details will be presented in a forthcoming publication [17].

\section{On-shell analysis of 2-, 3- and 4-body problem}

In a recent work [25] we show how using simple shell model variational calculations the binding energies of ${ }^{3} \mathrm{H},{ }^{4} \mathrm{He},{ }^{16} \mathrm{O}$ and ${ }^{40} \mathrm{Ca}$ nuclei can be reasonably well described by keeping the SRG evolved down to $\lambda \sim 1-2 \mathrm{fm}^{-1}$ two-body high quality potentials, i.e. interactions providing a $\chi^{2} /$ d.o.f $\lesssim 1$. In order to keep the numerical effort to a minimum and pursue the on-shell limit, $\lambda \rightarrow 0$, we use the gaussian separable toy model of Ref. [16] which describes the ${ }^{1} S_{0}$ and ${ }^{3} S_{1}$ channels reasonably well. Within such a scheme the energies are computed using $|t\rangle \equiv\left|(1 s)^{3}\right\rangle$ and $|\alpha\rangle \equiv\left|(1 s)^{4}\right\rangle$ with $1 s$ HO wave functions with a $b$ parameter,

$$
\left.\left\{E_{t}(\lambda), E_{\alpha}(\lambda)\right\}=\left\{-B_{t},-B_{\alpha}\right\}=\min _{b}\left[(A-1)\left\langle\frac{p^{2}}{2 M}\right\rangle_{1 s}+\frac{A(A-1)}{2} \frac{1}{2}\left\langle V_{1^{1}, \lambda}+V^{3} S_{1}, \lambda\right\rangle\right\rangle_{\mathrm{rel}, 1 s}\right]\left.\right|_{A=3,4}
$$

which can be interpreted in terms of the number of pairs in the ${ }^{1} S_{0}$ and ${ }^{3} S_{1}$ states being $n_{1} S_{0}, t=n_{3} S_{1} t=3 / 2$ for triton and $n_{1} S_{0}, \alpha=n_{3} S_{1}, \alpha=6 / 2$ for ${ }^{4} \mathrm{He}$. Within this shell model scheme $E_{d}(\lambda)=\min _{b}\left\langle p^{2} / M+V_{{ }^{3} S_{1}, \lambda}\right\rangle_{\text {rel }, 1 s}$. For $\lambda=\infty$ the deuteron is unbound by $0.2 \mathrm{MeV}$, and the binding energy obtained for the triton is $B_{t}^{\mathrm{Var}}=$ $5.9661 \mathrm{MeV}$ to be compared with the exact Faddeev equation result $B_{t}=6.65543 \mathrm{MeV}$ whereas the $\alpha$-particle yields $B_{\alpha}^{\mathrm{Var}}=32.1054 \mathrm{MeV}$.

It is interesting to analyze the SRG evolution from these $\lambda=\infty$ values down to $\lambda=0$ without 3-body or 4-body forces, i.e. taking $V_{3}(\lambda)=V_{4}(\lambda)=0$. One can show [17] that for the variational shell model

$$
\lim _{\lambda \rightarrow 0} E_{d}(\lambda)=-B_{d}, \quad \lim _{\lambda \rightarrow 0} E_{t}(\lambda)=-\frac{3}{2} B_{d}, \quad \lim _{\lambda \rightarrow 0} E_{\alpha}(\lambda)=-3 B_{d},
$$




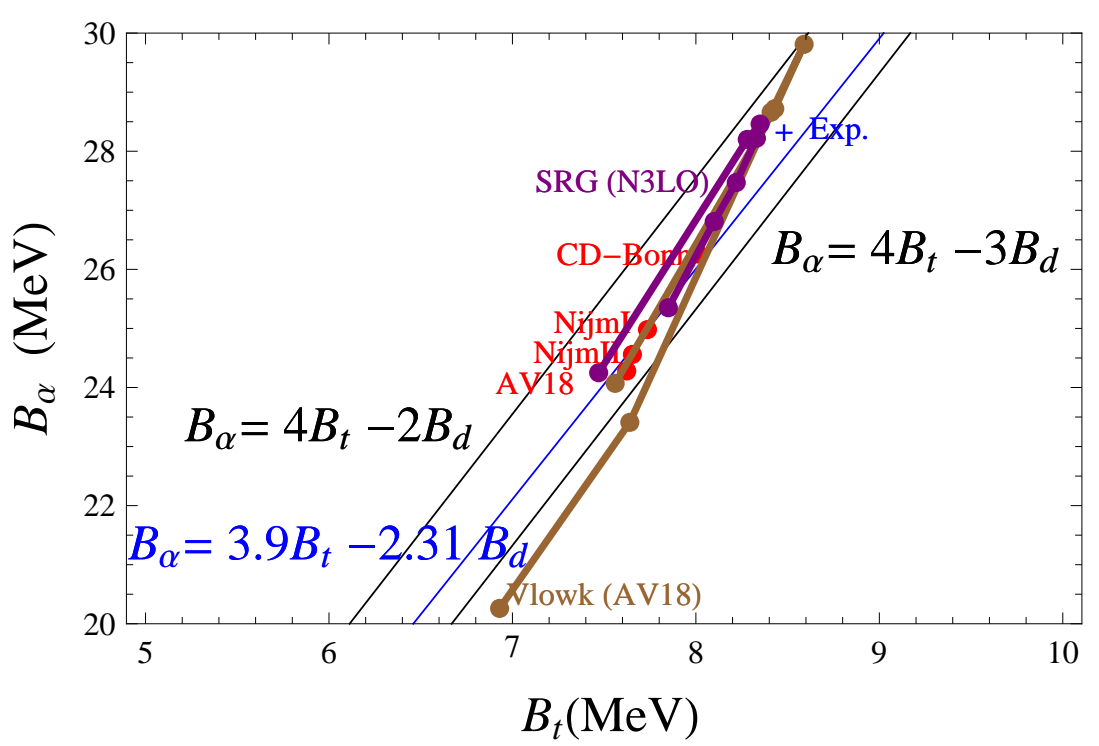

Fig. 2 Tjon line predicted by the on-shell 2-body interaction subjected to a 3-body force fulfilling $\left\langle V_{3}\right\rangle_{\alpha}=4\left\langle V_{3}\right\rangle_{t}$ but assuming different number of deuterons. We compare with few body calculations without 3-body forces [3] and 2-body high quality potentials fitting NN scattering data and the deuteron.

which is checked by the numerical calculation (see Fig. 11). We also find linear correlations in two regimes

$$
\Delta B_{\alpha} / \Delta B_{t} \sim 2(\lambda \rightarrow 0), \quad \Delta B_{\alpha} / \Delta B_{t} \sim 4(\lambda \sim 1) .
$$

If we now switch on the SRG induced 3-body and 4-body forces, the difference of the on-shell result to the original value corresponds to the off-shellnes of the $\lambda=\infty$ two body potential,

$$
-B_{t}=-\frac{3}{2} B_{d}+\left\langle t\left|V_{3}\right| t\right\rangle, \quad-B_{\alpha}=-3 B_{d}+\left\langle\alpha\left|V_{3}+V_{4}\right| \alpha\right\rangle .
$$

Neglecting $\left\langle\alpha\left|V_{4}\right| \alpha\right\rangle$ and taking $\left\langle\alpha\left|V_{3}\right| \alpha\right\rangle=4\left\langle t\left|V_{3}\right| t\right\rangle$ corresponding to 4 triplets in the $\alpha$-particle (see also [26]) we get $B_{\alpha}=4 B_{t}-3 B_{d}$ which gives $B_{\alpha}=4 \times 8.482-3 \times 2.225=27.53$ (exp.28.296) MeV. Of course, one may say the ${ }^{3} \mathrm{H}=$ pnn contain 2 deuterons and ${ }^{4} \mathrm{He}=$ ppnn contains 4 deuterons, so that $B_{\alpha}=4 B_{t}-2 B_{d}$. In general, we may write $n_{d / \alpha}=2 n_{3 S_{1}, \alpha}$ and $n_{d / t}=2 n_{3} S_{1, t}$ and hence

$$
B_{\alpha}=n_{t / \alpha} B_{t}+\left(n_{d / \alpha}-n_{d / t} n_{t / \alpha}\right) B_{d} / 2 .
$$

Most calculations in Nuclear Physics use a mean field reference state (often HO shell model) upon which correlations are built. For instance, in Ref. [27] it is found that the number of correlated pairs is given by $n_{1} S_{0, t}=1.490, n_{3} S_{1, t}=1.361, n_{1} S_{0}, \alpha=2.572$ and $n_{3 S_{1}, \alpha}=2.992$ with $\left(B_{d}, B_{t}, B_{\alpha}\right)=(2.24,7.76,25.09) \mathrm{MeV}$ which, from our Eq. $(10)$, requires $n_{t / \alpha}=3.9$ and then $B_{\alpha}=3.9 B_{t}-2.31 B_{d}$. These Tjon lines are plotted in Fig. 2 using the different choices in Eq. (10) and compared with several accurate calculations using realistic 2-body forces (without 3- or 4-body forces) [3]. In our interpretation, the slope of the Tjon line is just the number of correlated triplets in ${ }^{4} \mathrm{He},\left(\partial B_{\alpha} / \partial B_{t}\right)_{B_{d}}=n_{t / \alpha}=\left\langle V_{3}\right\rangle_{\alpha} /\left\langle V_{3}\right\rangle_{t}$ for on-shell interactions. These intriguing results suggest that $\left\langle\alpha\left|V_{4}\right| \alpha\right\rangle \lesssim 1 \mathrm{MeV}$ and will be analysed in more detail elsewhere [17].

\section{Conclusions}

SRG methods allow to reduce the two-body off-shell ambiguity completely when the infrared limit is taken and thus only measurable two-body information is needed. The same observation applies to multi-body interactions, and while current calculations fix the three body force from the triton binding energy, more work is required to pin down how much three-body measurable input would actually be demanded within such an on-shell scheme. However, using these ideas a simple explanation of the observed linear Tjon correlations between the $\alpha$-particle and triton binding energies emerges from purely combinatorial arguments. In this scheme the SRG evolved 3-body forces are large in the infrared limit whereas 4-body forces remain moderate. 


\section{References}

1. H. Ekstein: Equivalent Hamiltonians in scattering theory. Phys. Rev. 117, 1590 (1960)

2. W. Polyzou, W. Glöckle: Three-body interactions and on-shell equivalent two-body interactions. Few-Body Systems 9(2), 97 (1990)

3. H.W. Hammer, A. Nogga, A. Schwenk: Three-body forces: From cold atoms to nuclei. Rev.Mod.Phys. 85, 197 (2013)

4. A. Delfino, T. Frederico, V. Timóteo, L. Tomio: The few scales of nuclei and nuclear matter. Physics Letters B 634(2), 185 (2006)

5. S.D. Glazek, K.G. Wilson: Perturbative renormalization group for Hamiltonians. Phys. Rev. D49, 4214 (1994)

6. F. Wegner: Flow equations for Hamiltonians. Annalen der physik 506(2), 77 (1994)

7. S. Bogner, R. Furnstahl, R. Perry: Similarity Renormalization Group for Nucleon-Nucleon Interactions. Phys.Rev. C75, $061001(2006)$

8. V. Timoteo, S. Szpigel, E. Ruiz Arriola: Symmetries of the Similarity Renormalization Group for Nuclear Forces. Phys.Rev. C86, 034002 (2011)

9. S. Kehrein, The flow equation approach to many-particle systems (Springer, 2006)

10. S. Szpigel, R. Perry, A. Mitra, Quantum Field Theory, A 20th Century Profile (Hindustan Publishing Co., 2000)

11. S. Bogner, R. Furnstahl, R. Perry: Three-Body Forces Produced by a Similarity Renormalization Group Transformation in a Simple Model. Annals Phys. 323, 1478 (2007)

12. B.D. Jones, R.J. Perry: Similarity flow of a neutral scalar coupled to a fixed source. ArXiv:nucl-th/1305.6599 (2013)

13. S. Szpigel, V.S. Timoteo, F.d.O. Duraes: Similarity Renormalization Group Evolution of Chiral Effective Nucleon-Nucleon Potentials in the Subtracted Kernel Method Approach. Annals Phys. 326, 364 (2010)

14. W. Li, E. Anderson, R. Furnstahl: The Similarity Renormalization Group with Novel Generators. Phys.Rev. C84, 054002 (2011)

15. E. Anderson, S. Bogner, R. Furnstahl, E. Jurgenson, R. Perry, et al.: Block Diagonalization using SRG Flow Equations. Phys.Rev. C77, 037001 (2008)

16. E. Ruiz Arriola, S. Szpigel, V. Timoteo: Implicit vs Explicit Renormalization and Effective Interactions. Phys. Lett. B 728, $596(2014)$

17. E. Ruiz Arriola, S. Szpigel, V. Timoteo, In preparation (2013)

18. E. D. Jurgenson, P. Navratil and R. J. Furnstahl: Evolution of Nuclear Many-Body Forces with the Similarity Renormalization Group. Phys. Rev. Lett. 103 (2009) 082501

19. E. D. Jurgenson, P. Navratil and R. J. Furnstahl: Evolving Nuclear Many-Body Forces with the Similarity Renormalization Group, Phys. Rev. C 83, 034301 (2011)

20. K. Hebeler: Momentum space evolution of chiral three-nucleon forces. Phys. Rev. C 85, 021002 (2012)

21. E. D. Jurgenson, P. Maris, R. J. Furnstahl, P. Navratil, W. E. Ormand and J. P. Vary: P-shell nuclei using Similarity Renormalization Group evolved three-nucleon interactions, Phys. Rev. C 87, 054312 (2013)

22. K. A. Wendt: Similarity Renormalization Group Evolution of Three-Nucleon Forces in a Hyperspherical Momentum Representation. Phys. Rev. C 87, 061001 (2013)

23. R. J. Furnstahl and K. Hebeler: New applications of renormalization group methods in nuclear physics,” Rept. Prog. Phys. 76 (2013) 126301

24. R. Roth, A. Calci, J. Langhammer and S. Binder: Evolved Chiral NN+3N Hamiltonians for Ab Initio Nuclear Structure Calculations, arXiv:1311.3563 [nucl-th].

25. E. Ruiz Arriola, V. Timoteo, S. Szpigel: Nuclear Symmetries of the similarity renormalization group for nuclear forces. PoS CD12, 106 (2013)

26. M. Sato, Y. Akaishi, H. Tanaka: Effects of Three-Body Force in the Triton and the Alpha Particle. Prog. of Theor. Phys. Supplement, 56, 76 (1974)

27. H. Feldmeier, W. Horiuchi, T. Neff, Y. Suzuki: Universality of short-range nucleon-nucleon correlations. Phys.Rev. C84, $054003(2011)$ 